Atıf/Citation: R. G. TÜRECİ, "Lineer-Kuadratik Anizotropik Saçılma için Kritiklik Probleminde Yansıtıcı

Özdeğerleri”, Süleyman Demirel Üniversitesi Fen Edebiyat Fakültesi Fen Dergisi, 14, 1-15, 2019.

\title{
Lineer-Kuadratik Anizotropik Saçılma için Kritiklik Probleminde Yansıtıcı Özdeğerleri
}

\author{
Recep Gökhan TÜRECI ${ }^{1 *}$
}

${ }^{1}$ Kırıkkale Üniversitesi, Kırıkkale Meslek Yüksekokulu, 71450, Kırıkkale, Türkiye

*yazlşllan yazar e-posta: tureci@kku.edu.tr

Özet: Tek-hılı nötron transport teoride son zamanlarda çalışılan lineer-kuadratik anizotropik saçılma fonksiyonu belirli kritik levha kalınlıkları için yansıtıcı öz değerlerini çözmek amacıyla kullanılır. Lineer-kuadratik anizotropik saçılma özelliği gösteren nükleer materyale sahip bir düzlem reaktör göz önüne alınmıştır ve problem yansıtıcı sınır koşullarını içermektedir. Amaç belirli ikincil nötron sayılarına ve belirli reaktör kalınlıklarına göre yansıtıcı katsayılarını bulmaktır. Sayısal sonuçlar Modified $\mathrm{F}_{\mathrm{N}}$ yöntemi kullanılarak bulunmuştur.

Anahtar kelimeler: Lineer-kuadratik anizotropik saçılma, Case özfonksiyonları, Modified $\mathrm{F}_{\mathrm{N}}$ method, Kritiklik denklemi, Kritik kalınlık, Yansıtıcı özdeğerleri

\section{The Reflection Eigenvalues in the Criticality problem for Linear-Quadratic Anisotropic Scattering}

\begin{abstract}
The recently studied Linear-Quadratic anisotropic scattering function in one-speed neutron transport theory is used to solve the reflection eigenvalues for the certain critical slab thicknesses. A slab reactor including the nuclear material is taken into account for the linearquadratic anisotropic scattering with the reflection boundary condition. The aim is to find the reflection coefficients according to the secondary neutron numbers and the reactor thickness. The numerical results are obtained by using the Modified FN method.
\end{abstract}

Keywords: Linear-Quadratic anisotropic scattering, Case's eigenfunctions, Modified $\mathrm{F}_{\mathrm{N}}$ method, Criticality equation, Critical thickness, Reflection eigenvalues

\section{Giriş}

Nötron transport teori, nükleer materyalleri göz önüne alarak bir reaktör ortamı içindeki nötron dağılımını hesaplamak amacıyla kullanılır. Nötron yüksüz bir parçacık olduğundan Boltzmann denklemi olarak bilinen nötron transport denklemi, fotonlar göz önüne alınarak astrofizik ve optiksel oşinografi gibi alanlara da uygulanabilir.

Nötron transport denklemi üç boyutlu uzayda nötronların hızları, konumları ve zaman değişkeni de düşünüldüğünde yedi değişkene sahip bir denklemdir ve bu sebeple denklemi çözmek için belirli yaklaşımlarda bulunulur. Bu yaklaşımlar reaktörü oluşturan materyaller göz önüne alındığında makul yaklaşımlardır. Bir nötron kaynağından ya da bir nükleer tepkimeden ürün olarak açığa çıkan ikincil nötronlar belirli enerji değerlerine sahiptir. Bu nedenle tüm nötronların aynı enerjiye dolayısıyla da aynı hıza sahip olduğu tek-hızlı yaklaşım göz önüne alınır. Bir diğer yaklaşım, ortam olarak adlandırılan nükleer materyal bölgesinin homojen dağılıma sahip olması durumudur. Bu yaklaşımda da tesir kesitleri bir sabit olur ve bunun sonucunda da tesir kesiti oranı olan ikincil nötron sayısı 
sabit bir değerde olur. Bir diğer yaklaşım düzlem geometri yaklaşımıdır. Bu yaklaşımda birbirine paralel düzlemde nötron transport denkleminin çözümlerinin aynı olduğu düşünülür.

Bu yaklaşımlar altında düzlem geometride, tek-hızlı, homojen uzay, zamandan bağımsız nötron transport denklemi

$$
\mu \frac{\partial \Psi(x, \mu)}{\partial x}+\Psi(x, \mu)=\frac{c}{2} \int_{-1}^{1} f\left(\mu, \mu^{\prime}\right) \Psi\left(x, \mu^{\prime}\right) d \mu^{\prime}
$$

ile verilir [1-6]. Burada $\Psi(x, \mu), x$ noktası ve $\mu$ doğrultusundaki nötron akısı, $\mu$ nötronların ilerleme doğrultusunun kosinüsü, $\mu^{\prime}$ etkileşmeden sonra saçılan nötronların doğrultusunun kosinüsü, $c$ etkileşmeden sonra ortaya çıkan ikincil nötron sayısıdır. $f\left(\mu, \mu^{\prime}\right)$ nötronların saçılma olasılı̆̆ını tanımlayan saçılma fonksiyonudur. Saçılma fonksiyonu Mika [7] tarafından Legendre polinomları cinsinden seriye açılmıştır.

$$
f\left(\mu, \mu^{\prime}\right)=\sum_{n=0}^{M} f_{n}(2 n+1) P_{n}(\mu) P_{n}\left(\mu^{\prime}\right)
$$

Bu açılım ifadesinde $f_{n}$ saçılma katsayısı, $P_{n}(\mu)$ ve $P_{n}\left(\mu^{\prime}\right), n$. mertebeden Legendre polinomlarıdır. $\mathrm{Bu}$ saçılma fonksiyonunda $M=0$ ise $f_{0}$ terimi göz önüne alınır ve izotropik saçılmalı duruma karşı gelir. İzotropik saçılma durumunda nötronların her yönde saçılma olasılığı eşittir. Açılımda $M=1$ alınırsa bu durum lineer anizotropik saçılmaya karşı gelir. İzotropik saçılmaya ek olarak nötronların saçılma olasıllı̆̆ açısal değişken ile doğrusal olarak artar. Her iki saçılma durumu da farklı yöntemler ile çalışılmıştır; singüler özfonksiyonlar yöntemi [8,9], saçılma özdeğerleri [10], $F_{N}$ yöntemi [11,12], $\mathrm{C}_{\mathrm{N}}$ yöntemi [13] ve $\mathrm{H}_{\mathrm{N}}$ yöntemi [14]. $M=2$ seçimi $f_{1}=0$ koşulu ile safkuadratik anizotropik saçılmalı duruma [15-17], $M=3$ seçimi $f_{1}=0$ ve $f_{2}=0$ koşulu ile saf-triplet saçılmalı [18] durumlara karşı gelir.

Bu çalışmada lineer ve kuadratik saçılmalı durumlar birlikte düşünülmüş ve lineerkuadratik saçılma [19,20,21] olarak adlandırılmıştır. Dolayısıyla bu çalışmada saçılma fonksiyonu,

$$
f\left(\mu, \mu^{\prime}\right)=f_{0}+3 f_{1} P_{1}(\mu) P_{1}\left(\mu^{\prime}\right)+5 f_{2} P_{2}(\mu) P_{2}\left(\mu^{\prime}\right)
$$

olarak tanımlıdır. Eşitliğin sağ tarafındaki ilk terim izotropik saçılmaya, ikinci terim lineer anizotropik saçılmaya ve son terim de kuadratik anizotropik saçılmaya karşı gelir.

Fisyon tepkimelerinin gerçekleştiği bir düzlem reaktör göz önüne alınmaktadır. Böyle bir reaktörde Denklem (3) ile verilen lineer-kuadratik anizotropik saç1lma durumu için yansıtıcı sınır koşullarının da probleme dahil edilmesi ile kritiklik problemi ele alınmıştır. Kritiklik problemi, fisyon tepkimelerinden sonra açığa çıkan ikincil nötronlar ile düzlem reaktörün kalınlığı arasında matematiksel bir bağıntı bulabilme problemidir.

Denklem (3) ile verilen saçılma fonksiyonu için Denklem (1) ile verilen nötron transport denkleminin genel çözümü, 


$$
\begin{gathered}
\Psi(x, \mu)=A\left(v_{0}\right) \phi_{l q}\left(v_{0}, \mu\right) e^{-x / v_{0}}+A\left(-v_{0}\right) \phi_{l q}\left(-v_{0}, \mu\right) e^{x / v_{0}} \\
+\int_{-1}^{1} A(v) \phi_{l q}(v, \mu) e^{-x / v} d v, \mu \in[-1,1]
\end{gathered}
$$

olarak elde edilir. Denklem (4), izotropik saçılma için Case'in özfonksiyonlarını cinsinden yazılan genel çözüme benzer bir şekilde tanımlanmıştır. Burada özfonksiyonlar lineer-kuadratik anizotropik saçılma teriminin özelliklerini içinde barındırmaktadır. Denklem (4)' teki ilk terimde yer alan $\phi_{l q}\left(v_{0}, \mu\right), \quad+v_{0}$ özdeğerine karşı gelen özfonksiyon, ikinci terimde yer alan $\phi_{l q}\left(-v_{0}, \mu\right),-v_{0}$ özdeğerine karşı gelen özfonksiyon ve son terimdeki integral içerisinde yer alan $\phi_{l q}(v, \mu)$ sürekli özfonksiyondur. $A\left( \pm v_{0}\right)$ ve $A(v)$ terimleri ise keyfi açılım katsayılarıdır. Özfonksiyonları gösterirken kullanılan $l q$ alt indisi, bu özfonksiyonların lineer-kuadratik saçılmalı duruma ait olduğunu belirtmek için kullanılmıştır.

Lineer anizotropik saçılmalı durumlar için kesikli ve sürekli özfonksiyonlar ve bu özfonksiyonlar arasındaki diklik bağıntıları aşağıda verilmiştir.

Kesikli özfonksiyonlar,

$$
\phi_{l q}\left( \pm v_{0}, \mu\right)= \pm \frac{c v_{0}}{2} \frac{1-\eta_{0} \pm w v_{0} \mu+3 \eta \mu^{2}}{v_{0} \mp \mu}
$$

burada $\pm v_{0}$ kesikli özdeğerler olup diğer sabitler,

$$
\begin{gathered}
w=3 f_{1}(1-c) \\
\eta_{0} \equiv \eta\left(v_{0}\right)=\frac{5 f_{2}}{4}\left[3 v_{0}^{2}(1-c)\left(1-c f_{1}\right)-1\right]
\end{gathered}
$$

şeklinde tanımlıdır. Özdeğerler, Case özfonksiyonlarının bir sayısına normalize edildiği transandantal denklemden elde edilir:

$$
\begin{aligned}
\Lambda\left(v_{0}\right) & =1-\int_{-1}^{1} \phi_{l q}\left( \pm v_{0}, \mu\right) d \mu \\
= & \ln \left(\frac{1+1 / v_{0}}{1-1 / v_{0}}\right)-\frac{2}{c v_{0}} \frac{1+w c v_{0}^{2}+3 \eta_{0} c v_{0}^{2}}{1-\eta_{0}+w v_{0}^{2}+3 \eta_{0} v_{0}^{2}}=0
\end{aligned}
$$

Bu denklemin sayısal çözümleri $\pm v_{0}$ kesikli özdeğerleri verir. Sürekli özfonksiyon ise

$$
\begin{aligned}
& \phi_{l q}(v, \mu)=\frac{c v}{2} P \frac{1-\eta+w v \mu+3 \eta \mu^{2}}{v-\mu}+\lambda(v) \delta(v-\mu) \\
& \lambda(v)=\left(1+w c v^{2}+3 \eta c v^{2}\right)-c v\left(1-\eta+w v^{2}+3 \eta v^{2}\right) \operatorname{Arctan} h(v)
\end{aligned}
$$


ile verilir. Buradaki $P$ sembolü, $v=\mu$ durumunda ortaya çıkan singüler nokta etrafındaki Cauchy prensip değeri temsil etmektedir. Case özfonksiyonları aşağıdaki diklik bağıntılarına sağlarlar.

$$
\begin{aligned}
& \int_{-1}^{1} \mu \phi_{l q}\left( \pm v_{0}, \mu\right) \phi_{l q}\left( \pm v_{0}, \mu\right) d \mu=N_{l q}\left( \pm v_{0}\right) \text { ve } N_{l q}\left(-v_{0}\right)=-N_{l q}\left(v_{0}\right) \\
& \int_{-1}^{1} \mu \phi_{l q}(v, \mu) \phi_{l q}\left(v^{\prime}, \mu\right) d \mu=N_{l q}(v) \delta\left(v-v^{\prime}\right) \text { ve } N_{l q}(-v)=-N_{l q}(v)
\end{aligned}
$$

Burada

$$
\begin{aligned}
N_{l q}\left(v_{0}\right)=\left(\frac{c v_{0}}{2}\right)^{2}\left\{\frac{2 v_{0}}{v_{0}^{2}-1}\left[\left(1-\eta_{0}\right)^{2}+2\left(1-\eta_{0}\right) w v_{0}+\left(6 \eta_{0}\left(1-\eta_{0}\right)+w^{2} v_{0}^{2}\right)+6 w \eta_{0} v_{0}+9 \eta_{0}^{2}\right]\right. \\
-\left[\left(1-\eta_{0}\right)^{2}+4\left(1-\eta_{0}\right) w v_{0}^{2}+3 v_{0}^{2}\left(6 \eta_{0}\left(1-\eta_{0}\right)+w^{2} v_{0}^{2}\right)\right. \\
\left.+24 w \eta_{0} v_{0}^{4}+45 \eta^{2} v_{0}^{4}\right] \frac{2}{c v_{0}} \frac{1+w c v_{0}^{2}+3 \eta c v_{0}^{2}}{1-\eta_{0}+w v_{0}^{2}+3 \eta_{0} v_{0}^{2}} \\
+8\left(1-\eta_{0}\right) w v_{0}+6 v_{0}\left(6 \eta_{0}\left(1-\eta_{0}\right)+w^{2} v_{0}^{2}\right)+48 w \eta_{0} v_{0}^{3} \\
\left.+16 w \eta_{0} v_{0}+90 \eta_{0}{ }^{2} v_{0}^{3}+30 \eta_{0}{ }^{2} v_{0}\right\} \\
N_{l q}(v)=v\left[\left(1+w c v^{2}+3 \eta c v^{2}\right)-c v\left(1-\eta+w v^{2}+3 \eta v^{2}\right) \arctan h(v)\right]^{2} \\
+\frac{c^{2} \pi^{2} v^{3}}{4}\left(1-\eta+w v^{2}+3 \eta v^{2}\right)^{2}
\end{aligned}
$$

olarak tanımlıdır.

\section{Kritiklik Problemi}

Kritiklik probleminde amaç, ikincil nötron sayısı ile reaktörün kalınlığı arasında matematiksel bir bağıntı elde etmektir. Problem birçok araştırmacının üzerinde çalıştı̆̆ 1 sayısal çözümleme açısından zor bir problemdir [22-38]. Bu amaçla $x \in[-a, a]$ aralığında fisyon yapabilen nükleer materyalin bulunduğu düşünülür. Bu aralığın diş1 boşluk olarak adlandırılır. Bu tanımlama ile reaktör kalınlığı $\tau=2 a$ ' dır. Probleminde reaktörün $x=-a$ duvarı ile $x=a$ duvarından çıkan nötron akısının olduğu ve bu akıların simetrik olduğu kabul edilir. Böylece bu duvarlardan çıkan nötron akısı

$$
\Psi(-a,-\mu)=\Psi(a, \mu), \quad \mu \in[0,1]
$$

şeklinde tanımlanır ve simetri koşulu olarak adlandırılır. Matematiksel bir çözüm bulabilmek için çıkan nötron aklıları kuvvet serisi olarak tanımlanır.

$$
\Psi(-a,-\mu)=\Psi(a, \mu)=\sum_{\ell} a_{\ell} \mu^{\ell}, \quad \mu \in[0,1]
$$

Kritiklik probleminde boşluktan reaktöre, nötron girişi olmadığı düşünülür. Temel olarak fisyon sonucu üretilen ikincil nötronların ortamı terk etmesi istenir. Dolayısıyla giren nötron akıları sıfır kabul edilir. Ancak problemin daha gerçekçi olması için sınırdan belirli bir oranda nötronun yansıyarak ortama geri döndüğü düşünülebilir. Bu durumda kritiklik 
problemi, yansıtıcı sınır koşulu ile birlikte göz önüne alınır ve yansıtıcı katsayısının artması ile reaktör kalınlığının azalması beklenir.

Ortamdan içeri giren nötron akıları $R$ yansıma katsayısı cinsinden

$$
\begin{aligned}
& \Psi(-a, \mu)=R \Psi(-a,-\mu), \quad \mu \in[0,1] \\
& \Psi(a,-\mu)=R \Psi(a, \mu), \quad \mu \in[0,1]
\end{aligned}
$$

olarak tanımlanır. Burada $R$ yansıma katsayısıdır ve $R \in[0,1]$ aralığında tanımlıdır. Simetri koşulunun Denklem (4)' e uygulanmasıyla, keyfi açılım katsayıları arasında

$$
A\left(v_{0}\right)=A\left(-v_{0}\right) \text { ve } A(v)=A(-v)
$$

şeklinde bir ilişki olması gerektiği anlaşılır.

Bu aşamadan sonra Denklem (10-a) ve Denklem (10-b)' de verilen diklik bağıntılarının kullanılmasıyla açılım katsayıları sınır koşullarına bağlı olarak belirlenir. Bu düşünce Case, $F_{N}$ ve Modified $F_{N}\left(H_{N}\right)$ yöntemlerinde aynıdır. Yöntemlerin farklılığı katsayılar bulunduktan sonra yapılan işlemlerde ortaya çıkar. Katsayılar belirlendikten sonra, bilinen ikincil nötron sayıları için ortamın herhangi bir yerinde nötron akısı, açısal değişkene bağlı olarak biliniyor demektir.

Genel çözümde yer alan keyfi açılım katsayılarının belirlenmesi için, Denklem (4) ile verilen genel çözüm, reaktörün sağ duvarı yani $x=a$ sınırında yazılırsa

$$
\begin{aligned}
\Psi(a, \mu) & =A\left(v_{0}\right)\left[\phi_{l q}\left(v_{0}, \mu\right) e^{-a / v_{0}}+\phi_{l q}\left(-v_{0}, \mu\right) e^{a / v_{0}}\right] \\
& +\int_{0}^{1} A(v)\left[\phi_{l q}(\nu, \mu) e^{-a / v}+\phi_{l q}(-v, \mu) e^{a / v}\right] d v, \quad \mu \in[-1,1]
\end{aligned}
$$

Denklem (16) sırasıyla $\mu \phi_{l q}\left(-v_{0}, \mu\right)$ ve $\mu \phi_{l q}(-v, \mu)$ ile çarpılarak diklik bağıntılarını uygulamak için $\mu \in[-1,1]$ aralığında integre edilirse, açılım katsayıları

$$
\begin{aligned}
& A\left(v_{0}\right)=\frac{e^{-a / v_{0}}}{N\left(v_{0}\right)} \frac{c v_{0}}{2} \sum_{\ell} a_{\ell}\left[-A_{\ell}\left(v_{0}\right)+R B_{\ell}\left(v_{0}\right)\right] \\
& A(v)=\frac{e^{-a / v}}{N(v)} \frac{c v}{2} \sum_{\ell} a_{\ell}\left[-A_{\ell}(v)+R B_{\ell}(v)\right]
\end{aligned}
$$

olarak elde edilir. $\mathrm{Bu}$ ifadelerde yer alan $A_{\ell}(\xi)$ ve $B_{\ell}(\xi), \xi=v, v_{0}$ fonksiyonları, sayısal olarak hesaplanabilen ve lineer-kuadratik saçılmalı durum için aşağıdaki verilen şekliyle elde edilen özelliklere sahip fonksiyonlardır.

$$
A_{n}(\xi)=\frac{2}{c \xi} \int_{0}^{1} \mu^{n+1} \phi_{l q}(\xi,-\mu) d \mu, \quad \xi=v_{0} \text { or } v
$$




$$
\begin{aligned}
B_{n}(\xi)= & \frac{2}{c \xi} \int_{0}^{1} \mu^{n+1} \phi_{l q}(\xi, \mu) d \mu, \quad \xi=v_{0} \text { or } v \\
A_{n}(\xi)= & \frac{1-\zeta}{n+1}-\frac{w \xi}{n+2}+\frac{3 \zeta}{n+3}-\xi A_{n-1}(\xi) \\
A_{0}(\xi)= & 1-\zeta-\frac{w \xi(1-2 \xi)}{2}+\frac{\zeta}{2}\left(2-3 \xi+6 \xi^{2}\right) \\
-\xi\left(1-\zeta+w \xi^{2}+3 \zeta \xi^{2}\right) \ln \left(1+\frac{1}{\xi}\right) & \\
B_{n}(\xi)= & \xi B_{n-1}(\xi)-\frac{1-\zeta}{n+1}-\frac{w \xi}{n+2}-\frac{3 \zeta}{n+3} \\
B_{0}(\xi)= & \frac{2}{c}\left(1+c w \xi^{2}+3 c \zeta \xi^{2}\right)-(1-\zeta) \\
& -\frac{w \xi(1+2 \xi)}{2}-\frac{\zeta}{2}\left(2+3 \xi+6 \xi^{2}\right) \\
& -\xi\left(1-\zeta+w \xi^{2}+3 \zeta \xi^{2}\right) \ln \left(1+\frac{1}{\xi}\right)
\end{aligned}
$$

burada $\xi=v, v_{0}$ 'a ve $\zeta=\eta_{0}$ veya $\eta^{\prime}$ a karşı gelir.

Son olarak Denklem (17) ve Denklem (18), $x=a$ sınırından çıkan nötron akısı için yazılıp, $\mu^{m+1}$ ile çarpılarak $\mu \in[0,1]$ aralığında integre edilir ve aşağıda verilen denklem elde edilir.

$$
\begin{aligned}
\sum_{\ell} a_{\ell} & \left\{\frac{1}{m+\ell+2}+\left(\frac{c v_{0}}{2}\right)^{2} \frac{e^{-2 a / v_{0}}}{N\left(v_{0}\right)} B_{m}\left(v_{0}\right)\left[A_{\ell}\left(v_{0}\right)-R B_{\ell}\left(v_{0}\right)\right]\right. \\
& +\left(\frac{c v_{0}}{2}\right)^{2} \frac{A_{m}\left(v_{0}\right)}{N\left(v_{0}\right)}\left[A_{\ell}\left(v_{0}\right)-R B_{\ell}\left(v_{0}\right)\right] \\
& +\int_{0}^{1}\left(\frac{c v}{2}\right)^{2} \frac{e^{-2 a / v}}{N\left(v_{0}\right)} B_{m}(v)\left[A_{\ell}(v)-R B_{\ell}(v)\right] d v \\
& \left.+\int_{0}^{1}\left(\frac{c v}{2}\right)^{2} \frac{A_{m}(v)}{N\left(v_{0}\right)}\left[A_{\ell}(v)-R B_{\ell}(v)\right] d v\right\}=0
\end{aligned}
$$

Denklem

$$
\sum_{\ell} a_{\ell} T_{\ell m}=0
$$

şeklinde yazılabilir. Denklem (28)'deki $a_{\ell}$ katsayıları, ortamın sınırındaki nötron akısı için yazılan kuvvet serisinin katsayılardır.

Dolayısıyla bu katsayılar sıfır olamaz. Bu nedenle Denklem (28)' nin sağlanabilmesi için elemanları $T_{\ell m}$ olan matrisin determinantı sıfir olmalıdır: 
Denklem (29) kritiklik denklemidir ve verilen ikincil nötron sayısı ve kritik kalınlık değerlerine göre yansıtıcı katsayılarını belirlemek için çözülmüştür. Bu şekliyle Denklem (29) yansitıcı katsayısının lineer bir fonksiyonudur.

\section{Sayısal sonuçlar}

Tablolarda yer alan sayısal sonuçlar Denklem (29)' un çözümünden elde edilmiştir. Sayısal çözümler, Mathematica v13.1 programı ve Newton-Raphson yöntemi kullanılarak yapılmıştır.

Denklem (3) ile verilen saçılma fonksiyonu aynı zamanda nötronların saçılma olasılığını tanımlar ve olasılıkların toplamı 1 olmak zorunda olduğundan lineer anizotropik saçılma katsayısı ile kuadratik anizotropik saçılma katsayısı arasında lineer bağımlılık söz konusudur. Bu lineer bağımlılık

$$
\frac{3 f_{1}-1}{5} \leq f_{2} \leq \frac{3 f_{1}}{5}
$$

olarak elde edilmiştir. Dolayısıyla lineer anizotropik saçılma katsayısı $f_{1}$ ' in alacağı değerlere göre kuadratik anizotropik saçılma katsayısı $f_{2}$ ' nin alacağı değerler sınırlıdır ve Denklem (30) ile tanımlanır.

Denklem (29) yansıtıcı özdeğerlerinin aranan değişken olarak düşünüldüğü durumda yansitıcı özdeğeri $R$ ' nin lineer bir fonksiyonudur. Kritiklik problemi ikincil nötron sayısının kritik 1 değerinden büyük olduğu durumlar için incelenir. İkincil nötron sayısının 1' den büyük olduğu durumda kesikli özdeğerler kompleks sayılardır.

Tablo $1, c=1.1$ ve $c=1.7$ için belirli $f_{1}$ değerlerine karşılık $f_{2}$ ' nin alabileceği değerleri ve bu aralıktan seçilen $f_{2}$ değerlerine göre kesikli özdeğerleri göstermektedir. Tablo 2, $c=1.1$ için sirasiyla $f_{1}=-0.3,-0.2,-0.1,0.1$ için ve Tablo $3, c=1.1$ için sırasıyla $f_{1}=0.2,0.3$ için belirli kritik kalınlık değerleri için yansıtıcı özdeğerlerini göstermektedir. Tablo 4, değişen ikincil nötron sayısı, saçılma katsayıları ve yansıtıcı katsayılarına göre hesaplanmış kritik kalınlık değerlerini göstermektedir [39]. Sonuçların birbiri ile tutarlı olduğu görülmektedir. 
Tablo 1. $c=1.1$ ve $c=1.7$ değerleri ve belirli $f_{1}$ değerlerine karşı gelen $f_{2}$ değerleri için elde edilen kesikli özdeğerler

\begin{tabular}{cccccc}
\hline \multicolumn{5}{c}{$c=1.1$} \\
\hline$f_{1}=-0.1 \Rightarrow-0.26 \leq f_{2} \leq-0.06$ & $f_{1}=-0.2 \Rightarrow-0.32 \leq f_{2} \leq-0.12$ & $f_{1}=-0.3 \Rightarrow-0.38 \leq f_{2} \leq-0.18$ \\
& & & & & \\
\hline$f_{2}$ & $\pm i v_{0}$ & $f_{2}$ & $\pm i v_{0}$ & $f_{2}$ & $\pm i v_{0}$ \\
\hline-0.26 & 1.681559264668154 & -0.32 & 1.606488489015353 & -0.38 & 1.540801414304454 \\
& & & & & \\
-0.2 & 1.678928308026901 & -0.3 & 1.605761390132237 & -0.32 & 1.538855572374482 \\
-0.1 & 1.673891447375443 & -0.2 & 1.601757058747913 & -0.3 & 1.538166722231152 \\
-0.05 & 1.671005217548424 & -0.12 & 1.598031116175141 & -0.2 & 1.534375246546119
\end{tabular}

\begin{tabular}{cccccc}
\hline \multicolumn{2}{c}{$f_{1}=0.1 \Rightarrow-0.14 \leq f_{2} \leq 0.06$} & \multicolumn{2}{c}{$f_{1}=0.2 \Rightarrow-0.08 \leq f_{2} \leq 0.12$} & \multicolumn{2}{c}{$f_{1}=0.3 \Rightarrow-0.02 \leq f_{2} \leq 0.18$} \\
\hline$f_{2}$ & $\pm i v_{0}$ & $f_{2}$ & $\pm i v_{0}$ & $f_{2}$ & $\pm i v_{0}$ \\
\hline-0.14 & 1.870904526044534 & -0.08 & 1.993980409770264 & -0.02 & 2.145903805493660 \\
& & & & & \\
-0.1 & 1.868468316110832 & -0.05 & 1.991781724223652 & 0.05 & 2.139267654463619 \\
& & & & & \\
0.05 & 1.865152051472937 & 0.1 & 1.978471843780226 & 0.1 & 2.133855468731677 \\
0.06 & 1.856562505644721 & 0.12 & 1.976335980718684 & 0.18 & 2.123728914828428 \\
\hline \multicolumn{5}{c}{$c=1.7$} \\
\hline$f_{1}=-0.1 \Rightarrow-0.26 \leq f_{2} \leq-0.06$ & $f_{1}=-0.2 \Rightarrow-0.32 \leq f_{2} \leq-0.12$ & $f_{1}=-0.3 \Rightarrow-0.38 \leq f_{2} \leq-0.18$
\end{tabular}

\begin{tabular}{cccccc}
\hline$f_{2}$ & $\pm i v_{0}$ & $f_{2}$ & $\pm i v_{0}$ & $f_{2}$ & $\pm i v_{0}$ \\
\hline-0.26 & 0.541595714198719 & -0.32 & 0.512807097392654 & -0.38 & 0.488450044486201 \\
-0.2 & 0.536410253877516 & -0.3 & 0.511461029213720 & -0.32 & 0.485038068693529 \\
-0.1 & 0.526557542588061 & -0.2 & 0.504085279570762 & -0.3 & 0.483834346831312 \\
-0.05 & 0.520973380157779 & -0.12 & 0.497296180094156 & -0.2 & 0.477255316798761
\end{tabular}

\begin{tabular}{cccccc}
\hline \multicolumn{2}{c}{$f_{1}=0.1 \Rightarrow-0.14 \leq f_{2} \leq 0.06$} & \multicolumn{2}{c}{$f_{1}=0.2 \Rightarrow-0.08 \leq f_{2} \leq 0.12$} & \multicolumn{2}{c}{$f_{1}=0.3 \Rightarrow-0.02 \leq f_{2} \leq 0.18$} \\
\hline$f_{2}$ & $\pm i v_{0}$ & $f_{2}$ & $\pm i v_{0}$ & $f_{2}$ & $\pm i v_{0}$ \\
\hline-0.14 & 0.620064827917168 & -0.08 & 0.677258940995195 & -0.02 & 0.757998317746030 \\
& & & & & \\
-0.1 & 0.614309257802116 & -0.05 & 0.671319816028993 & 0.05 & 0.736359646405465 \\
& & & & & \\
0.05 & 0.606491565611146 & 0.1 & 0.635626570265981 & 0.1 & 0.718764611670485 \\
0.06 & 0.586458897486417 & 0.12 & 0.629997796703203 & 0.18 & 0.686410995322673 \\
\hline
\end{tabular}

Tablo 5-10, $c=2.0$ için lineer anizotropik saçılma katsayısının değerlerine ve kuadratik anizotropik saçılma katsayılarına göre hesaplanmış değerleri göstermektedir.

Tablo 2. $c=1.1$ ve değişen $f_{1}$ ve kritik kalınlıklar için yansıtıcı özdeğerleri

\begin{tabular}{cccccc}
\hline \multicolumn{5}{c}{$c=1.1, \quad f_{1}=-0.3$ ve $f_{2}=-0.38$} \\
\hline$N$ & $\tau=0.5$ & $\tau=1$ & $\tau=2$ & $\tau=3$ & $\tau=3.8$ \\
\hline 1 & 0.900558 & 0.806239 & 0.610042 & 0.358502 & 0.037733 \\
2 & 0.901639 & 0.807189 & 0.608837 & 0.352754 & 0.027944 \\
3 & 0.901636 & 0.807189 & 0.608803 & 0.352720 & 0.028025 \\
4 & 0.901637 & 0.807188 & 0.608803 & 0.352723 & 0.028029
\end{tabular}




\begin{tabular}{|c|c|c|c|c|c|}
\hline 5 & 0.901636 & 0.807187 & 0.608803 & 0.352723 & .02002 \\
\hline \multicolumn{6}{|c|}{$c=1.1, f_{1}=-0.3$ ve $f_{2}=-0.2$} \\
\hline 1 & 0.900032 & 0.805270 & 0.6080272 & 0.354385 & 0.029034 \\
\hline 2 & 0.901319 & 0.806542 & 0.6071865 & 0.348805 & 0.019170 \\
\hline 3 & 0.901319 & 0.806540 & 0.6071380 & 0.348759 & 0.019292 \\
\hline 4 & 0.901320 & 0.806539 & 0.6071390 & 0.348764 & 0.019297 \\
\hline 5 & 0.901320 & 0.806539 & 0.607139 & 0.348764 & 0.019297 \\
\hline \multicolumn{6}{|c|}{$c=1.1, \quad f_{1}=-0.2$ ve $f_{2}=-0.32$} \\
\hline 1 & 0.900413 & 0.8064269 & 0.613886 & 0.376523 & .093392 \\
\hline 2 & 0.901609 & 0.8075124 & 0.61 & 0.37 & \\
\hline 3 & 0.901608 & 0.8075131 & 0.61 & 0.3 & 530 \\
\hline 4 & 0.901608 & 0.8075119 & 0.61 & 0.37 & 533 \\
\hline 5 & 07 & 0.8075114 & 0.612755 & 0.37 & \\
\hline \multicolumn{6}{|c|}{$c=1.1, \quad f_{1}=-0.2$ ve $f_{2}=-0.12$} \\
\hline 1 & & 0.805205 & 0.611408 & 0.371 & \\
\hline 2 & 0.9 & 0.806711 & 0.61 & 0.36 & \\
\hline 3 & 0.901213 & 0.806709 & 0.61 & 0.36 & \\
\hline 4 & 0.90 & 0.806708 & 0.61 & & \\
\hline 5 & & 708 & & 0.36 & \\
\hline \multicolumn{6}{|c|}{$c=1.1, \quad f_{1}=-0.1$ ve $f_{2}=-0.26$} \\
\hline$N$ & $\tau=0.5$ & $\tau=1$ & $\tau=2$ & $\tau=3$ & $\tau=4$ \\
\hline 1 & 0.900238 & 0.806570 & $0.61^{\prime}$ & 0.39 & 059411 \\
\hline 2 & 0. & 317 & 0.61 & 0.3 & 693 \\
\hline 3 & 0.9 & 818 & 0.61 & 0.3 & 0.0 \\
\hline 4 & 0.9 & 0.807816 & 0.61 & 0.38 & 792 \\
\hline 5 & & 0.807816 & 0.616567 & 0.387 & \\
\hline \multicolumn{6}{|c|}{$c=1.1, \quad f_{1}=-0.1$ ve $f_{2}=-0.05$} \\
\hline 1 & & 14 & 0.61 & 0.3 & \\
\hline 2 & & & & & \\
\hline 3 & 0.9 & 78 & 0.6 & 0.3 & \\
\hline 4 & 06 & 877 & 0.6 & 0.3 & \\
\hline 5 & 66 & 0.806876 & 0.614266 & 0.38 & 37191 \\
\hline \multicolumn{6}{|c|}{$c=1.1, \quad f_{1}=0.1$ ve $f_{2}=-0.14$} \\
\hline 1 & & 0.806681 & 0.624466 & 0.42 & 2029 \\
\hline 2 & 0.9 & 354 & 0.62 & 0.41 & 1171 \\
\hline 3 & 0.901461 & 8356 & 0.62 & 0.4 & 1238 \\
\hline 4 & & 354 & 0.62 & & \\
\hline 5 & 0.901460 & 0.808354 & 0.623770 & 0.418260 & 51242 \\
\hline \multicolumn{6}{|c|}{$c=1.1, \quad f_{1}=0.1$ ve $f_{2}=-0.06$} \\
\hline 1 & 784 & 0.804902 & 0.621111 & 0.417839 & 1779 \\
\hline 2 & 0.900909 & 0.807261 & 0.621250 & 0.41 & 0.140810 \\
\hline 3 & 0.900914 & 0.807260 & 0.621196 & 0.413090 & 0.140920 \\
\hline 4 & 0.900914 & 0.807258 & 0.621196 & 0.413094 & 0.140925 \\
\hline 5 & 0.900914 & 0.807257 & 0.621196 & 0.413094 & 0.140925 \\
\hline
\end{tabular}

Tablo 3. $c=1.1$ ve $f_{1}=0.2,0.3$ için değişen kritik kalınlıklar için yansıtıcı özdeğerleri

\begin{tabular}{|c|c|c|c|c|c|}
\hline \multicolumn{6}{|c|}{$c=1.1, \quad f_{1}=0.2$ ve $f_{2}=-0.08$} \\
\hline$N$ & $\tau=0.5$ & $\tau=1$ & $\tau=1.323$ & $\tau=3.78$ & $\tau=4.6189$ \\
\hline 1 & 0.899447 & 0.806618 & 0.748595 & 0.261185 & 0.013628 \\
\hline 2 & 0.901383 & 0.808581 & 0.750077 & 0.251610 & 0.000152 \\
\hline 3 & 0.901386 & 0.808584 & 0.750068 & 0.251641 & 0.000327 \\
\hline 4 & 0.901386 & 0.808581 & 0.750066 & 0.251644 & 0.000331 \\
\hline 5 & 0.901385 & 0.808581 & 0.750066 & 0.251644 & 0.000331 \\
\hline \multicolumn{6}{|c|}{$c=1.1, \quad f_{1}=0.2$ ve $f_{2}=0.12$} \\
\hline$N$ & $\tau=0.5$ & $\tau=1$ & $\tau=2$ & $\tau=4$ & $\tau=4.5$ \\
\hline 1 & 0.898306 & 0.804560 & 0.623857 & 0.193634 & 0.040364 \\
\hline
\end{tabular}




\begin{tabular}{|c|c|c|c|c|c|}
\hline 2 & 0.900761 & 0.807351 & 0.624377 & 0.182755 & 0.026828 \\
\hline 3 & 0.900768 & 0.807351 & 0.624322 & 0.182853 & 0.027069 \\
\hline 4 & 0.900768 & 0.807349 & 0.624323 & 0.182859 & 0.027074 \\
\hline 5 & 0.900767 & 0.807348 & 0.624323 & 0.182859 & 0.027074 \\
\hline \multicolumn{6}{|c|}{$c=1.1, \quad f_{1}=0.3$ ve $f_{2}=-0.02$} \\
\hline$N$ & $\tau=0.5$ & $\tau=1$ & $\tau=2.71$ & $\tau=4$ & $\tau=4.84547562$ \\
\hline 1 & 0.899046 & 0.806444 & 0.503978 & 0.240918 & 0.014550 \\
\hline 2 & 0.901290 & 0.808774 & 0.500567 & 0.230099 & $|-0.000196|$ \\
\hline 3 & 0.901294 & 0.808777 & 0.500530 & 0.230151 & $|-3.160349| \cdot 10^{-6} \rightarrow 0$ \\
\hline 4 & 0.901294 & 0.808774 & 0.500532 & 0.230155 & $|-7.536247| \cdot 10^{-8} \rightarrow 0$ \\
\hline 5 & 0.901293 & 0.808774 & 0.500532 & 0.230155 & $1.780576 \cdot 10^{-8} \rightarrow 0$ \\
\hline \multicolumn{6}{|c|}{$c=1.1, \quad f_{1}=0.3$ ve $f_{2}=0.18$} \\
\hline$N$ & $\tau=0.1$ & $\tau=0.5$ & $\tau=0.50307641$ & $\tau=1$ & $\tau=4$ \\
\hline 1 & 0.978445 & 0.897703 & 0.897106 & 0.804032 & 0.230429 \\
\hline 2 & 0.979424 & 0.900581 & 0.899993 & 0.807380 & 0.219675 \\
\hline 3 & 0.979424 & 0.900590 & 0.900001 & 0.807380 & 0.219766 \\
\hline 4 & 0.979424 & 0.900590 & 0.900002 & 0.807377 & 0.219771 \\
\hline 5 & 0.979424 & 0.900589 & 0.900001 & 0.807376 & 0.219771 \\
\hline
\end{tabular}

Tablo 4. Ref.[39]'dan seçilmiş $c, f_{1}, f_{2}$ ve $R$ değerlerine göre hesaplanmış kritik kalınlık değerleri

\begin{tabular}{ccccclc}
\hline & \multicolumn{1}{c}{$c=1.1}$, & $f_{1}=0.2$ ve $f_{2}=-0.08$ & $c=1.1$, & $f_{1}=0.3 \quad f_{2}=-0.02$ & $c=1.1$, & $f_{1}=0.3 \quad f_{2}=0.18$ \\
\hline$N$ & $R=0.75$ & $R=0.25$ & $R=0.0$ & $R=0.0$ & $R=0.9$ \\
\hline 1 & 1.315209 & 3.823893 & 4.656906 & 4.891355 & 0.488053 \\
2 & 1.323425 & 3.786264 & 4.619330 & 4.844851 & 0.503033 \\
3 & 1.323378 & 3.786386 & 4.619825 & 4.845465 & 0.503080 \\
4 & 1.323364 & 3.786400 & 4.619834 & 4.845475 & 0.503080 \\
5 & 1.323362 & 3.786400 & 4.619834 & 4.845476 & 0.503076 \\
\hline
\end{tabular}

Tablo 5. $c=2.0$ ve $f_{1}=-0.3$ için değişen $f_{2}$ ve kritik kalınlıklar için yansıtıcı özdeğerleri

\begin{tabular}{|c|c|c|c|c|c|}
\hline \multicolumn{6}{|c|}{$c=2.0, \quad f_{1}=-0.3$} \\
\hline \multicolumn{6}{|c|}{$f_{2}=-0.38$} \\
\hline$N$ & $\tau=0.05$ & $\tau=0.1$ & $\tau=0.3$ & $\tau=0.4$ & $\tau=0.5$ \\
\hline 1 & 0.885994 & 0.781635 & 0.433738 & 0.286438 & 0.148420 \\
\hline 2 & 0.887871 & 0.786433 & 0.449845 & 0.305957 & 0.169630 \\
\hline 3 & 0.888405 & 0.787559 & 0.452487 & 0.308642 & 0.171943 \\
\hline 4 & 0.888447 & 0.787656 & 0.452569 & 0.308585 & 0.171716 \\
\hline 5 & 0.888441 & 0.787654 & 0.452554 & 0.308556 & 0.171684 \\
\hline \multicolumn{6}{|c|}{$f_{2}=-0.32$} \\
\hline 1 & 0.885462 & 0.780443 & 0.429599 & 0.280810 & 0.141275 \\
\hline 2 & 0.886967 & 0.784803 & 0.446138 & 0.301364 & 0.164093 \\
\hline 3 & 0.887596 & 0.786084 & 0.449001 & 0.304240 & 0.166534 \\
\hline 4 & 0.887648 & 0.786196 & 0.449088 & 0.304169 & 0.166274 \\
\hline 5 & 0.887642 & 0.786194 & 0.449071 & 0.304136 & 0.166237 \\
\hline \multicolumn{6}{|c|}{$f_{2}=-0.3$} \\
\hline 1 & 0.885285 & 0.780040 & 0.428150 & 0.278824 & 0.138738 \\
\hline 2 & 0.886644 & 0.784222 & 0.444817 & 0.299729 & 0.162120 \\
\hline 3 & 0.887307 & 0.785558 & 0.447761 & 0.302673 & 0.164607 \\
\hline 4 & 0.887363 & 0.785676 & 0.447849 & 0.302597 & 0.164335 \\
\hline 5 & 0.887356 & 0.785674 & 0.447831 & 0.302562 & 0.164295 \\
\hline \multicolumn{6}{|c|}{$f_{2}=-0.2$} \\
\hline 1 & 0.884436 & 0.778016 & 0.420371 & 0.267985 & 0.124723 \\
\hline 2 & 0.884849 & 0.780988 & 0.437482 & 0.290643 & 0.151154 \\
\hline 3 & 0.885703 & 0.782636 & 0.440872 & 0.293967 & 0.153894 \\
\hline 4 & 0.885779 & 0.782787 & 0.440969 & 0.293862 & 0.153548 \\
\hline 5 & 0.885773 & 0.782786 & 0.440945 & 0.293817 & 0.153498 \\
\hline
\end{tabular}

Tablo 6. $c=2.0$ ve $f_{1}=-0.2$ için değişen $f_{2}$ ve kritik kalınlıklar için yansıtıcı özdeğerleri

\begin{tabular}{c}
$c=2.0, \quad f_{1}=-0.2$ \\
\hline$f_{2}=-0.32$
\end{tabular}




\begin{tabular}{cccccc}
\hline$N$ & $\tau=0.05$ & $\tau=0.1$ & $\tau=0.3$ & $\tau=0.4$ & $\tau=0.5$ \\
\hline 1 & 0.885113 & 0.779920 & 0.430830 & 0.284775 & 0.149546 \\
2 & 0.887032 & 0.784981 & 0.448468 & 0.306342 & 0.173191 \\
3 & 0.887627 & 0.786216 & 0.451276 & 0.309162 & 0.175588 \\
4 & 0.887664 & 0.786308 & 0.451355 & 0.309100 & 0.175356 \\
5 & 0.887657 & 0.786304 & 0.451343 & 0.309075 & 0.175331 \\
\hline & & $f_{2}=-0.3$ & \\
\hline 1 & 0.884928 & 0.779502 & 0.429359 & 0.282777 & 0.147022 \\
2 & 0.886709 & 0.784399 & 0.447153 & 0.304722 & 0.171252 \\
3 & 0.887338 & 0.785691 & 0.450042 & 0.307611 & 0.173695 \\
4 & 0.887379 & 0.785789 & 0.450123 & 0.307545 & 0.173452 \\
5 & 0.887371 & 0.785785 & 0.450110 & 0.307519 & 0.173425 \\
\hline & & & & \\
\hline 1 & 0.884035 & $f_{2}=-0.2$ & 0.271876 & 0.133082 \\
2 & 0.884909 & 0.777399 & 0.421457 & 0.295717 & 0.160465 \\
3 & 0.885735 & 0.781160 & 0.439845 & 0.298997 & 0.163168 \\
4 & 0.885796 & 0.782773 & 0.443193 & 0.298902 & 0.162857 \\
5 & 0.885789 & 0.782902 & 0.443283 & 0.298868 & 0.162821 \\
\hline & & 0.782899 & 0.443265 & & \\
\hline 1 & 0.883417 & & $f_{2}=-0.12$ & & 0.120119 \\
2 & 0.883206 & 0.775789 & 0.414486 & 0.261960 & 0.150284 \\
3 & 0.884222 & 0.778098 & 0.432954 & 0.287227 & 0.153231 \\
4 & 0.884304 & 0.780022 & 0.436745 & 0.290880 & 0.152803 \\
5 & 0.884297 & 0.780183 & 0.436843 & 0.290756 & \\
\hline
\end{tabular}

Tablo 7. $c=2.0$ ve $f_{1}=-0.1$ için değişen $f_{2}$ ve kritik kalınlıklar için yansıtıcı özdeğerleri

\begin{tabular}{|c|c|c|c|c|c|}
\hline \multicolumn{6}{|c|}{$c=2.0, \quad f_{1}=-0.1$} \\
\hline \multicolumn{6}{|c|}{$f_{2}=-0.26$} \\
\hline$N$ & $\tau=0.05$ & $\tau=0.1$ & $\tau=0.3$ & $\tau=0.4$ & $\tau=0.5$ \\
\hline 1 & 0.884138 & 0.778001 & 0.427281 & 0.282270 & 0.149600 \\
\hline 2 & 0.886105 & 0.783370 & 0.446731 & 0.306266 & 0.176130 \\
\hline 3 & 0.886765 & 0.784722 & 0.449722 & 0.309233 & 0.178617 \\
\hline 4 & 0.886796 & 0.784808 & 0.449797 & 0.309167 & 0.178383 \\
\hline 5 & 0.886787 & 0.784803 & 0.449788 & 0.309148 & 0.178365 \\
\hline \multicolumn{6}{|c|}{$f_{2}=-0.2$} \\
\hline 1 & 0.883578 & 0.776679 & 0.422330 & 0.275475 & 0.140976 \\
\hline 2 & 0.884984 & 0.781353 & 0.442208 & 0.300721 & 0.169535 \\
\hline 3 & 0.885771 & 0.782913 & 0.445494 & 0.303938 & 0.172188 \\
\hline 4 & 0.885813 & 0.783017 & 0.445574 & 0.303855 & 0.171915 \\
\hline 5 & 0.885804 & 0.783012 & 0.445562 & 0.303832 & 0.171893 \\
\hline \multicolumn{6}{|c|}{$f_{2}=-0.1$} \\
\hline 1 & 0.882778 & 0.774594 & 0.413357 & 0.262792 & 0.124532 \\
\hline 2 & 0.882804 & 0.777438 & 0.433448 & 0.289981 & 0.156747 \\
\hline 3 & 0.883843 & 0.779410 & 0.437318 & 0.293690 & 0.159723 \\
\hline 4 & 0.883911 & 0.779553 & 0.437408 & 0.293572 & 0.159364 \\
\hline 5 & 0.883901 & 0.779549 & 0.437391 & 0.293540 & 0.159332 \\
\hline \multicolumn{6}{|c|}{$f_{2}=-0.05$} \\
\hline 1 & 0.882503 & 0.773705 & 0.408598 & 0.255798 & 0.115220 \\
\hline 2 & 0.881537 & 0.775166 & 0.428379 & 0.283766 & 0.149338 \\
\hline 3 & 0.882727 & 0.777383 & 0.432595 & 0.287765 & 0.152501 \\
\hline 4 & 0.882811 & 0.777551 & 0.432691 & 0.287624 & 0.152087 \\
\hline 5 & 0.882801 & 0.777547 & 0.432671 & 0.287586 & 0.152048 \\
\hline
\end{tabular}

Tablo 8. $c=2.0$ ve $f_{1}=0.1$ için değişen $f_{2}$ ve kritik kalınlıklar için yansıtıcı özdeğerleri

\begin{tabular}{cccccc}
\hline \multicolumn{7}{c}{$c=2.0}$, & $f_{1}=0.1$ \\
& & \multicolumn{3}{c}{$f_{2}=-0.14$} & \\
\hline$N$ & $\tau=0.05$ & $\tau=0.1$ & $\tau=0.3$ & $\tau=0.4$ & $\tau=0.5$ \\
\hline 1 & 0.881793 & 0.773307 & 0.417508 & 0.273794 & 0.145427 \\
2 & 0.883941 & 0.779578 & 0.441960 & 0.304468 & 0.179858 \\
3 & 0.884742 & 0.781191 & 0.445368 & 0.307769 & 0.182545 \\
4 & 0.884750 & 0.781250 & 0.445430 & 0.307697 & 0.182316
\end{tabular}




\begin{tabular}{|c|c|c|c|c|c|}
\hline 5 & 0.884738 & 0.781242 & 0.445428 & 0.307691 & 0.182317 \\
\hline \multicolumn{6}{|c|}{$f_{2}=-0.1$} \\
\hline 1 & 0.881433 & 0.772373 & 0.413564 & 0.268295 & 0.138418 \\
\hline 2 & 0.883012 & 0.777911 & 0.438270 & 0.299987 & 0.174593 \\
\hline 3 & 0.883929 & 0.779714 & 0.441946 & 0.303513 & 0.177428 \\
\hline 4 & 0.883946 & 0.779787 & 0.442013 & 0.303428 & 0.177169 \\
\hline 5 & 0.883934 & 0.779779 & 0.442009 & 0.303421 & 0.177169 \\
\hline \multicolumn{6}{|c|}{$f_{2}=-0.05$} \\
\hline 1 & 0.881433 & 0.772373 & 0.413564 & 0.268295 & 0.138418 \\
\hline 2 & 0.883012 & 0.777911 & 0.438270 & 0.299987 & 0.174593 \\
\hline 3 & 0.883929 & 0.779714 & 0.441946 & 0.303513 & 0.177428 \\
\hline 4 & 0.883946 & 0.779787 & 0.442013 & 0.303428 & 0.177169 \\
\hline 5 & 0.883934 & 0.779779 & 0.442009 & 0.303421 & 0.177169 \\
\hline \multicolumn{6}{|c|}{$f_{2}=0.06$} \\
\hline 1 & 0.880990 & 0.770043 & 0.397387 & 0.243934 & 0.105739 \\
\hline 2 & 0.878381 & 0.769625 & 0.420028 & 0.277833 & 0.148511 \\
\hline 3 & 0.879911 & 0.772429 & 0.425096 & 0.282515 & 0.152089 \\
\hline 4 & 0.879982 & 0.772587 & 0.425185 & 0.282355 & 0.151647 \\
\hline 5 & 0.879968 & 0.772578 & 0.425175 & 0.282335 & 0.151636 \\
\hline
\end{tabular}

Tablo 9. $c=2.0$ ve $f_{1}=0.2$ için değişen $f_{2}$ ve kritik kalınlıklar için yansıtıcı özdeğerleri

\begin{tabular}{|c|c|c|c|c|c|}
\hline \multicolumn{6}{|c|}{$c=2.0, \quad f_{1}=0.2$} \\
\hline \multicolumn{6}{|c|}{$f_{2}=-0.08$} \\
\hline$N$ & $\tau=0.05$ & $\tau=0.1$ & $\tau=0.3$ & $\tau=0.4$ & $\tau=0.5$ \\
\hline 1 & 0.880295 & 0.770266 & 0.410535 & 0.266907 & 0.140174 \\
\hline 2 & 0.882685 & 0.777358 & 0.438806 & 0.302591 & 0.180467 \\
\hline 3 & 0.883548 & 0.779096 & 0.442429 & 0.306062 & 0.183249 \\
\hline 4 & 0.883536 & 0.779129 & 0.442482 & 0.305992 & 0.183036 \\
\hline 5 & 0.883522 & 0.779120 & 0.442483 & 0.305993 & 0.183048 \\
\hline \multicolumn{6}{|c|}{$f_{2}=-0.05$} \\
\hline 1 & 0.880049 & 0.769567 & 0.407279 & 0.262308 & 0.134281 \\
\hline 2 & 0.881901 & 0.775952 & 0.435717 & 0.298856 & 0.176103 \\
\hline 3 & 0.882869 & 0.777862 & 0.439581 & 0.302529 & 0.179017 \\
\hline 4 & 0.882864 & 0.777907 & 0.439637 & 0.302449 & 0.178780 \\
\hline 5 & 0.882849 & 0.777897 & 0.439639 & 0.302450 & 0.178792 \\
\hline \multicolumn{6}{|c|}{$f_{2}=0.1$} \\
\hline 1 & 0.886081 & 0.772347 & 0.392594 & 0.238346 & 0.101246 \\
\hline 2 & 0.878670 & 0.769263 & 0.419139 & 0.278325 & 0.151612 \\
\hline 3 & 0.879170 & 0.770842 & 0.422776 & 0.281570 & 0.153779 \\
\hline 4 & 0.879195 & 0.771113 & 0.423289 & 0.281842 & 0.153738 \\
\hline 5 & 0.879232 & 0.771139 & 0.423243 & 0.281803 & 0.153738 \\
\hline \multicolumn{6}{|c|}{$f_{2}=0.12$} \\
\hline 1 & 0.897741 & 0.780809 & 0.392731 & 0.235888 & 0.096716 \\
\hline 2 & 0.879519 & 0.770361 & 0.419664 & 0.278287 & 0.150887 \\
\hline 3 & 0.878980 & 0.770364 & 0.421062 & 0.279299 & 0.150930 \\
\hline 4 & 0.878926 & 0.770652 & 0.421995 & 0.280025 & 0.151301 \\
\hline 5 & 0.878945 & 0.770640 & 0.421869 & 0.279924 & 0.151277 \\
\hline
\end{tabular}

Tablo 10. $c=2.0$ ve $f_{1}=0.3$ için değişen $f_{2}$ ve kritik kalınlıklar için yansıtıcı özdeğerleri

\begin{tabular}{cccccc}
\hline \multicolumn{5}{c}{$c=2.0, \quad f_{1}=0.3$} \\
\hline$N$ & $\tau=0.05$ & $\tau=0.1$ & $f_{2}=-0.02$ & \\
\hline 1 & 0.878316 & 0.766250 & 0.400883 & 0.256726 & 0.131130 \\
2 & 0.881322 & 0.774925 & 0.435080 & 0.299961 & 0.180099 \\
3 & 0.882209 & 0.776734 & 0.438865 & 0.303557 & 0.182938 \\
4 & 0.882165 & 0.776726 & 0.438903 & 0.303496 & 0.182760 \\
5 & 0.882148 & 0.776715 & 0.438909 & 0.303505 & 0.182782 \\
\hline & & \multicolumn{5}{c}{0.4} & \\
\hline 1 & 0.878787 & 0.765276 & 0.392089 & 0.243885 & 0.114445 \\
2 & 0.879753 & 0.771704 & 0.427297 & 0.290419 & 0.168847 \\
3 & 0.880371 & 0.773328 & 0.430990 & 0.293840 & 0.171367 \\
4 & 0.880300 & 0.773379 & 0.431194 & 0.293906 & 0.171258 \\
5 & 0.880385 & 0.773446 & 0.431209 & 0.293924 & 0.171298 \\
\hline
\end{tabular}




\begin{tabular}{|c|c|c|c|c|c|}
\hline \multicolumn{6}{|c|}{$f_{2}=0.1$} \\
\hline 1 & 0.895788 & 0.774846 & 0.384014 & 0.231045 & 0.098146 \\
\hline 2 & 0.882302 & 0.774964 & 0.429503 & 0.291382 & 0.168317 \\
\hline 3 & 0.879931 & 0.771967 & 0.426277 & 0.287887 & 0.164274 \\
\hline 4 & 0.879574 & 0.771978 & 0.427469 & 0.289029 & 0.165148 \\
\hline 5 & 0.879700 & 0.772110 & 0.427474 & 0.289063 & 0.165267 \\
\hline \multicolumn{6}{|c|}{$f_{2}=0.18$} \\
\hline 1 & 0.964054 & 0.825288 & 0.390474 & 0.224625 & 0.081813 \\
\hline 2 & 0.883566 & 0.777430 & 0.432157 & 0.292478 & 0.167113 \\
\hline 3 & 0.879938 & 0.771895 & 0.423992 & 0.283737 & 0.157834 \\
\hline 4 & 0.879572 & 0.771841 & 0.425390 & 0.285184 & 0.159012 \\
\hline 5 & 0.879632 & 0.771898 & 0.425364 & 0.285193 & 0.159110 \\
\hline
\end{tabular}

\section{Sonuç ve Yorum}

Tablolara aktarılan değerlere göre şu sonuçlara varılır:

- Artan $f_{1}$ değerlerine karşılık sabit tutulan $f_{2}$ değerleri için yansıma katsayısı artmaktadir.

- Sabit tutulan $f_{1}$ değerleri için artan $f_{2}$ değerleri ile yansıma katsayısı düşmektedir.

- Artan ikincil nötron sayısına bağlı olarak kritik kalınlık değerleri düşmektedir. İkincil nötron sayısının artışındaki etki yanında kritik kalınlık değeri arttıkça yansıtıcı katsayısının değeri düşmektedir.

Bu sonuçlar $f_{1}$ ve $f_{2}$ arasında kurulan lineer bağımlılık durumuna göre yapılmıştır. Farklı bir incelemede lineer bağımlılık $f_{1}$ yerine $f_{2}$ ' ye göre yapılabilirdi. Fakat açısal değişken $\mu \in[0,1]$ arasında değer aldığında açısal değişkenin kuadratik terimlerinin saçılmaya daha az etkisi olacağı düşünülmüştür.

Tablo 4, farklı ikincil nötron sayısı, saçılma katsayıları ve yansıtıcı katsayıları için kritiklik kalınlık değerlerini göstermektedir. Bu tablodaki değerler, Tablolar 1, 2 ve 3' ten seçilmiş bazı değerler için gösterilmiştir ve sonuçların tutarlı olduğu görülmektedir. İki çalışma arasındaki fark, Denklem (29) ile verilen kritiklik denkleminde hangi değişken için çözüm yapılması ile ilgilidir. Bu çalışmada kritiklik denklemi yansıtıcı katsayısının bir lineer fonksiyonudur. Fakat kritik kalınlık hesabının yapıldığı çalışmalarda, üstel terimde yer alan kritik kalınlık için çözüm yapılmaktadır. Özellikle integral terimi içerisinde yer alan üstel ifadenin çözümü oldukça zordur. Denklem (29)' u yansitıcı katsayısı için sayısal olarak çözmek çok daha kolay bir hesaplama sağlamaktadır.

Case yöntemi, yöntemin kendisinden gelen $\mathrm{X}$-fonksiyonlarının hesaplanmasındaki güçlük nedeniyle zor bir yöntemdir. Nispeten daha pratik olan $F_{N}$ yöntemi ile Modified $\mathrm{F}_{\mathrm{N}}\left(\mathrm{H}_{\mathrm{N}}\right)$ yöntemi arasında, denklem sistemi elde etme aşamasında farklılık vardır. Her iki yöntemde Case özfonksiyonlarının kullanımına dayanan yöntemlerdir. $F_{N}$ yönteminde genel çözümdeki katsayılar, problemin sınır şartlarına göre belirlenerek genel çözümde yazıldıktan sonra, elde edilen ifade matematiksel olarak aralıklara bölünür ve bölünen aralık sayısına göre denklem sistemi elde edilir. Modified $F_{N}\left(H_{N}\right)$ yönteminde ise katsayılar belirlenerek, genel çözümde yazıldıktan sonra, ifade $\mu^{m+1}$ ile çarpılarak integre edilir. Böylece kuvvet serisinin mertebesi ve kuvvet serisinin mertebesiyle birlikte değişen $m$ sayısına göre bir denklem sistemi elde edilir. Sayısal sonuçlar açısından Modified $\mathrm{F}_{\mathrm{N}}$ yöntemi $\mathrm{F}_{\mathrm{N}}$ yönteminden daha başarılı bir yöntemdir. 


\section{Kaynakça}

[1] B. Davison, Neutron Transport Theory. London: Oxford University Press, 1958, ch. 1-8.

[2] K. M. Case, "Elementary solutions of the transport equation and their applications," Ann. Physics, vol. 9, no. 1 , pp. 1-23, 1960.

[3] K. M. Case, and P. F. Zweifel, Linear Transport Theory. Reading Mass: Addition-Wesley, 1967, ch. 4-6.

[4] M. M. R. Williams, Mathematical Methods in Particle Transport Theory. New York: WileyInterscience, 1971, ch. 3-8.

[5] G. I. Bell, and S. Glasstone, Nuclear Reactor Theory. New York: Von Nostrand Reinhold, 1972, ch. $1-3$.

[6] W. M. Stacey, Neutron Transport Theory. 2nd ed., Weinheim: Wiley-VCH Verlag GmbH \& Co. KGaA, 2007. ch. 9.

[7] J. Mika, "Neutron transport with anisotropic scattering," Nucl. Sci. Eng., vol. 11, no. 4, pp. 415-427, 1961.

[8] N. J. McCormick, and I. Kuščer, "Half-space neutron transport with linearly anisotropic scattering," Journal of Mathematical Physics, vol. 6, no. 2, pp. 1939-1945, 1965.

[9] N. J. McCormick, and I. Kuščer, "Singular eigenfunction expansions in neutron transport theory," Advances in Nuclear Science and Technology, vol. 7, pp. 181-282, 1973.

[10] V. Protopopescu, and N. G. Sjöstrand, "On the solution of the dispersion equation for monoenergetic neutron transport with linearly anisotropic scattering," Prog. Nucl. Energ., vol. 7, no. 1, pp. 47-58, 1981.

[11] C. E. Siewert, and P. Benoist, "The $F_{N}$ method in neutron-transport theory, Part I: Theory and applications," Nucl. Sci. Eng., vol. 69, no. 2, pp. 156-160, 1979.

[12] P. Grandjean, and C. E. Siewert, "The F $F_{N}$ method in neutron-transport theory, Part II: Applications and numerical results,” Nucl. Sci. Eng., vol. 69, no. 2, pp. 161-168, 1979.

[13] A. Kavenoky, "The CN method of solving the transport equation: Application to plane geometry," Nucl. Sci. Eng., vol. 65, no. 2, pp. 209-225, 1978.

[14] C. Tezcan, A. Kaşkaş, M. Ç. Güleçyüz, "The H-N method for solving linear transport equation: theory and applications," J. Quant. Spectrosc. Ra., vol. 78, no. 2, pp. 243-254, 2003.

[15] N. G. Sjöstrand, "Complex eigenvalues for neutron transport equation with quadratically anisotropic scattering," J. Nucl. Sci. Technol., vol. 18, no. 1, pp. 1-5, 1981.

[16] R. G. Türeci, and D. Türeci, "Time dependent albedo problem for quadratic anisotropic scattering," Kerntechnik, vol. 72, no. 1-2, pp. 59-65, 2007.

[17] R. G. Türeci, and M.Ç. Güleçyüz, "The slab albedo and criticality problem for the quadratic scattering kernel with the H-N method," Kerntechnik, vol. 73, no. 4, pp. 171-175, 2008.

[18] R. G. Türeci, "Solving the criticality problem with the reflected boundary condition for the triplet anisotropic scattering with the Modified $\mathrm{F}_{\mathrm{N}}$ method," Kerntechnik, vol. 80, no. 6, pp. 583-591, 2015.

[19] R. G. Türeci, and D. Türeci, "Half-space albedo problem with Modified $F_{N}$ method for linear and quadratic anisotropic scattering," Kerntechnik, vol. 82, no. 2, pp. 239-245, 2017.

[20] R. G. Türeci, and D. Türeci, "Slab albedo for linearly and quadratically anisotropic scattering kernel with Modified F $F_{N}$ method" Kerntechnik, vol. 82, no.5, pp. 605-611, 2017.

[21] R. G. Türeci, "Half-space albedo problem for the (İnönü, Linear and Quadratic) anisotropic scattering," Kerntechnik, submitted for publication.

[22] G. J. Mitsis, "Transport solutions to the one-dimensional critical problem," Nucl. Sci. Eng., vol. 17, no. 1, pp. 55-64, 1963.

[23] I. Carlvik, "Monoenergetic critical parameters and decay constants for small homogeneous spheres and thin homogeneous slabs," Nucl. Sci. Eng., vol. 31, no. 2, pp. 295-303, 1968.

[24] D. C. Sahni, and N. G. Sjöstrand, "Criticality and time eigenvalues for one-speed neutron transport," Prog. Nucl. Energ., vol. 23, no. 3, pp.241-289, 1990.

[25] D. C. Sahni, N. G. Sjöstrand, and N. S. Garis, "Criticality and time eigenvalues for one-speed neutron in slab with forward and backward scattering," J. Phys. D. Appl. Phys., vol. 25 no. 10, pp. 1381-1389, 1992.

[26] D. C. Sahni, "Some new results pertaining to criticality and time eigenvalues of one-speed neutron transport equation," Prog. Nucl. Energ., vol. 30, no. 3, pp.305-320, 1996.

[27] C. E. Siewert, and M. M. R. Williams, "The effect of anisotropic scattering on the critical slab problem in neutron transport theory using a synthetic kernel," J. Phys. D. Appl. Phys., vol. 10, no. 15, pp. 2031$2040,1977$.

[28] E. B. Dahl, V. Protopopescu, and N. G. Sjöstrand, "On the relation between decay constants and critical parameters in monoenergetic neutron transport," Nucl. Sci. Eng., vol. 83, no. 3, pp. 374-379, 1983.

[29] C. Tezcan, and R. Sever, "The critical slab with the backward-forward-isotropic scattering model," Ann. Nucl. Energy., vol. 12, no.10, pp. 573-576, 1985.

[30] C. Tezcan, and C. Y1ldiz, "The criticality problems with the $\mathrm{F}_{\mathrm{N}}$ method for the FBIS model," Ann. Nucl. Energy., vol. 13, no. 6, pp. 345-348, 1986. 
[31] D. C. Sahni, and N. G. Sjöstrand, "Criticality and time eigenvalues in one-speed neutron transport," Prog. Nucl. Energ., vol. 23, no. 3, pp. 241-289, 1990.

[32] N. S. Garis, "One-speed neutron transport eigenvalues for reflected slabs and spheres," Nucl. Sci. Eng., vol. 107, no. 4, pp. 343-358, 1991.

[33] C. Tezcan, and C. Y1ld1z, "The transformation of $P_{L}$ solutions for extremely anisotropic scattering to solutions with isotropic scattering and application to the critical slab problem," Journal of Quantative Spectroscopy and Radiative Transfer, vol. 49, no. 4, pp. 411-416, 1993.

[34] M. A. Atalay, "The critical slab problem for reflecting boundary conditions in one-speed neutron transport theory," Ann. Nucl. Energy., vol. 23, no. 3, pp. 183-193, 1996.

[35] M. A. Atalay, "The reflected slab and sphere criticality problem with anisotropic scattering in one spedd neutron transport theory," Prog. Nucl. Energ., vol. 31, no. 3, pp. 229-252, 1997.

[36] R. G. Türeci, M. Ç. Güleçyüz, A. Kaşkaş, and C. Tezcan, "Application of the H-N method to the critical slab problem for reflecting boundary conditions," Journal of Quantative Spectroscopy and Radiative Transfer, vol. 88, no. 4, pp. 499-517, 2004.

[37] R. G. Türeci, M. Ç. Güleçyüz, A. Kaşkaş, C. Tezcan, "The singular eigenfunction method: the critical slab problem for linearly anisotropic scattering," Kerntechnik, vol. 70, no. 5-6, pp. 322-326, 2005.

[38] M. Ç. Güleçyüz, R.G. Türeci, and C. Tezcan, "The critical slab problem for linearly anisotropic scattering and reflecting boundary conditions with the H-N method," Kerntechnik, vol. 71, no. 1-2, pp.149-154, 2006.

[39] R. G. Türeci, "The criticality problem with linear-quadratic anisotropic scattering for reflected boundary condition," Kerntechnik, submitted for publication. 